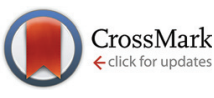

Cite this: Org. Biomol. Chem., 2016, 14, 7606

Received 1st June 2016,

Accepted 5th July 2016

DOI: $10.1039 / c 6 o b 01193 d$

www.rsc.org/obc

\title{
The role of boronic acids in accelerating condensation reactions of $\alpha$-effect amines with carbonyls
}

\begin{abstract}
Dennis Gillingham
A broad palette of bioconjugation reactions are available for chemical biologists, but an area that still requires investigation is high-rate constant reactions. These are indispensable in certain applications, particularly for in vivo labelling. Appropriately positioned boronic acids accelerate normally sluggish Schiff base condensations of $\alpha$-effect nucleophiles by five orders of magnitude - providing a new entry to the rare set of reactions that have a rate constant above $100 \mathrm{M}^{-1} \mathrm{~s}^{-1}$ under physiological conditions. I summarize here a number of recent reports, including work from my own group, and outline a mechanistic picture that explains the differing behaviour of seemingly similar substrate classes.
\end{abstract}

\section{Introduction}

Bioorthogonal ligation reactions ${ }^{1}$ that operate in water at neutral $\mathrm{pH}$ and have high second order rate constants are rare and yet serve important niches of chemical biology. ${ }^{2,3}$ A recent article from the Bode group outlines in detail the specific scenarios where high rate constants are necessary ${ }^{2}$ - I recap here the main points:

- if one or both components are precious then running under pseudo-first order conditions to achieve a high-rate may not be viable

- components with a high molecular weight (proteins, nucleic acids, PEG units) simply cannot be dissolved at high molar concentration

- large excesses of reagent in live cell or whole animal experiments often lead to confounding effects

- for fluorescent labelling large excesses of a reagent give high background signal or require additional washing steps before imaging

- high-rate constants reduce the necessary incubation times.

Recently my group has shown that the venerable oxime condensation can be accelerated by more than five orders of magnitude by including an appropriately positioned boronic acid in the carbonyl substrate. ${ }^{4}$ Others have demonstrated that this effect holds with other types of $\alpha$-effect amine condensations. ${ }^{5}$ In this Perspective I have collected the key results from previous reports on boron-accelerated condensations and attempted to synthesize this into a mechanistic picture that explains the special role of the boron atom in the process.

Department of Chemistry, University of Basel, St. Johanns-Ring 19, 4056, Basel, Switzerland. E-mail: dennis.gillingham@unibas.ch
The inverse-electron demand Diels-Alder reaction of strained olefins with tetrazines (TIEDDA) is currently the fastest ligation reaction used in chemical biology with second order rate constants upwards of $10^{6} \mathrm{M}^{-1} \mathrm{~s}^{-1}$ for optimized substrate pairs. ${ }^{6}$ The success of the TIEDDA reaction notwithstanding, having a handful of such transformations would be helpful in, for example, dual-labelling experiments, ${ }^{7}$ where two orthogonal reactions are required, or in cases where the disadvantages of the Diels-Alder reaction preclude its application (when the footprint interferes with function, or when the substrate syntheses are too demanding).

\section{Discussion}

The oxime condensation is a workhorse in chemical biology because the starting materials are readily available and the reaction is extremely reliable. ${ }^{8}$ Its rate, however, is slow with a maximum between $\mathrm{pH} 4$ and $5 .{ }^{9}$ For such condensations of $\alpha$-effect amines ( $O$-alkylhydroxylamines, hydrazines, hydrazones) with carbonyls Jencks had shown that aniline could serve as a nucleophilic catalyst. ${ }^{10}$ Dawson developed this idea further to create the aniline catalysis protocol, ${ }^{11}$ which is widely used for oxime/hydrazone ligations in chemical biology. With aniline catalysis oxime and hydrazone condensations can proceed with rate constants of up to $8 \mathrm{M}^{-1} \mathrm{~s}^{-1} \cdot{ }^{12}$ But the requirement for a catalyst (typically at high concentration) inherently reduces the scope of the catalysis approach to in vitro or cell surface labelling. ${ }^{13}$

There are few small molecule ligation reactions that operate under physiological conditions with second order rate constants above $100 \mathrm{M}^{-1} \mathrm{~s}^{-1} \cdot{ }^{14}$ Two processes that meet these 
standards - and consequently are widely used in chemical biology - are the TIEDDA reaction with strained olefins and certain variants of the copper catalysed azide-alkyne cycloaddition (CuAAC). ${ }^{15,16}$ Aside from the disadvantage of requiring an added catalyst, the CuAAC reaction is also hamstrung by the cellular toxicity of copper. ${ }^{17}$ Over the past seven years since its rediscovery as a reaction in chemical biology, the TIEDDA process with strained olefins has proven to be robust and broadly applicable, even being used for imaging in whole animals. ${ }^{18}$ Despite the complexity (and sometimes instability) of the reaction partners and the large chemical footprint left by this reaction, it is broadly used because of its combination of speed, chemoselectivity, and bioorthogonality. ${ }^{18-21}$ While the TIEDDA reaction has pushed the boundaries of what's possible in chemical biology, it has not supplanted oxime condensations and CuAAC reactions for day-to-day couplings because these reactions are easy to engineer, sterically lean, and the starting materials can be stored without decomposition. The ideal would be for the simplest reactions (oxime condensation, CuAAC reaction) to also have enzyme-like rate constants. While ligand refinement or copper chelation seem most promising for optimizing CuAAC reactions, ${ }^{15,22,23}$ it is the precise positioning of boronic acids in the carbonyl substrate that has elevated oxime and hydrazone condensations into rate regimes of up to $10^{4} \mathrm{M}^{-1} \mathrm{~s}^{-1}$. 4,5

While there were scattered reports that boron could lead to stabilization of normally labile Schiff bases, ${ }^{24-27}$ it was two reports in 2012 that pointed towards the potential of this effect in chemical biology. ${ }^{28,29}$ These demonstrated that boronic acids positioned near the carbonyl group led to stable Schiff bases with amines in whole proteins ${ }^{28}$ or in aminoglycoside antibiotics $^{29}$ in aqueous buffer. Recent work has applied boronate-stabilized Schiff base formation to the labelling of cell surface amines in bacteria, ${ }^{30}$ and in the reversible formation of cyclic peptides. ${ }^{31}$ In 2015 my group ${ }^{4}$ and the Gao lab ${ }^{5}$ published almost simultaneously that $\alpha$-effect amines condensed with boronic acid-bearing carbonyl substrates at rates far above normal oxime condensations. My group focussed on oximes formed from 2-formylphenylboronic acid (2-FPBA) while the Gao lab took a broader look at the $\alpha$-effect amines with 2-acetylphenylboronic acid (2-APBA). Taken together the results from my lab and the Gao lab suggest that 2-FPBA adducts are more stable and better suited to bioconjugation, while 2-APBA adducts are more labile and better suited to applications like dynamic combinatorial chemistry.

To understand the impact of boronic acids on these condensations it is instructive to compare different scenarios with and without boronic acids. In normal Schiff base formation there is a rapid addition equilibrium of the nucleophile to the carbonyl followed by a dehydration step (often rate-limiting) to give the Schiff base (see A in Fig. 1). ${ }^{9}$ Both steps are reversible and in water the Schiff base is disfavoured by le Chatelier's principle applied on the relatively small equilibrium constant for imines (typically $<100 \mathrm{M}^{-1}$ ). ${ }^{32}$ With boronic acids several effects likely stabilize the Schiff bases: in structure 4 (Fig. 1) both the hydrogen bond and the coulombic interaction

\section{A Typical Schiff base formation}
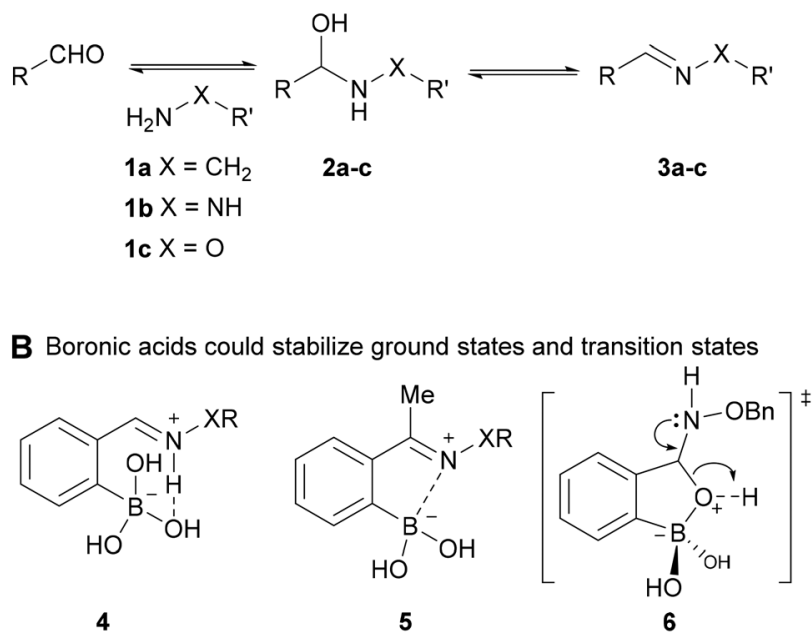

Fig. 1 Schiff base formations (A) and the impact that boronic acids might have on their ground states and transition states (B).

operate in concert (as is well-established with pyridoxyl phosphate-derived imines) ${ }^{32}$ to stabilize the Schiff base relative to the benzaldehyde imine - this mode of stabilization turns out to be most prevalent with 2-FPBA derived imines. ${ }^{29}$ With 2-APBA imines, on the other hand, calculations suggest that a Lewis acid/base interaction to form an iminoboronate is the key stabilizing force. ${ }^{28}$ Although they are more stable than normal imines, structures related to 5 have low kinetic barriers and re-equilibrate in seconds upon perturbation irrespective of whether they are imines, ${ }^{28}$ hydrazones, ${ }^{5}$ or oximes. ${ }^{33}$ For the ground states, however, it has so far not been determined whether structures such as $\mathbf{4}$ or $\mathbf{5}$ in the hydrazone or oxime series are more stable than the parent derivatives lacking a boronic acid (such as $\mathbf{3 b}$-c). A crystal structure of a boronic acid bearing oxime provides the only insight, and suggests that at least in the case of oximes the iminoboronate structure is not a substantial contributor to ground state energies of aldoximes. $^{33}$

How does boronic acid substitution lead to such a large change in kinetic barriers? The addition equilibrium in all types of Schiff base formation is typically rapid and hence the major change is likely in the dehydration step. Boronic acids are in an equilibrium with the boronate form in water and the indirect $\mathrm{p} K_{\mathrm{a}}$ associated with water addition is approximately 7 depending on substitution. For example the hemiaminal equilibrium shown in the bottom of Fig. 2 has a $\mathrm{p} K_{\mathrm{a}}$ of $7.4 .^{34}$ Coming back to oxime condensations, upon addition of a nucleophile the resulting tetrahedral intermediate can substitute for a water on the boron to give a cyclic boronate (as shown in structure 6 of panel B in Fig. 1). In a normal oxime condensation the $O$-alkylhydroxylamine is more basic than the alcohol lone pair in the hemiaminal intermediate (compare competing transition states 7 \& 8 in Fig. 2); hence transition state $\mathbf{8}$ is preferred and the forward reaction is correspondingly slower. When boron is present the competition is now the 


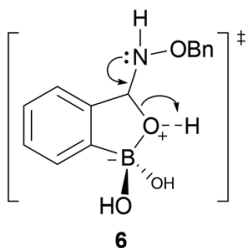<smiles>[B]ONC(C)(O)c1ccccc1</smiles>

7
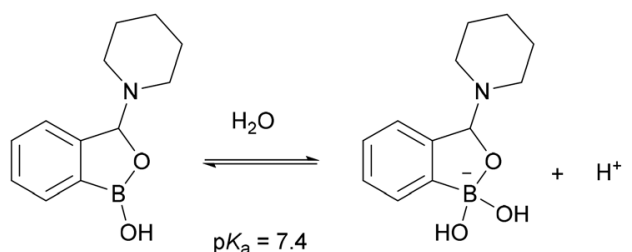

Fig. 2 Top: Comparing normal oxime formation with the boron-accelerated variant; Bottom: Boronate formation causes the oxygen lone pair to have an unusually high $\mathrm{p} K_{\mathrm{a}}$, which is essential for the elimination reaction shown in structure 6 .

boronate versus the protonated $O$-alkylhydroxylamine as the leaving group. The preference for boronate elimination favors productive oxime formation (see 6 in Fig. 2) leading to faster rates than in the parent oxime formation. The direct involvement of the vacant boron p-orbital is further supported by experiments where tripodal ligands or fluoride substituents on the boron abolished the rate acceleration. ${ }^{33}$

A disadvantage of the boron-assisted Schiff base formations is their reversibility. The aldoxime formations with 2 -formylphenylboronic acid that we have reported give the most kinetically stable adducts but even these have a reverse rate constant of $4.2 \pm 0.4 \times 10^{-5} \mathrm{~s}^{-1}$, meaning adducts can be hydrolysed completely in about half a day. Oxime condensations with 2FPBA do, however, have large equilibrium constant of $>10^{8}$ $\mathbf{M}^{-1}$, and this mitigates the reversibility problem since even at nanomolar concentration of starting materials the oxime will predominate at equilibrium. Nevertheless for cellular studies
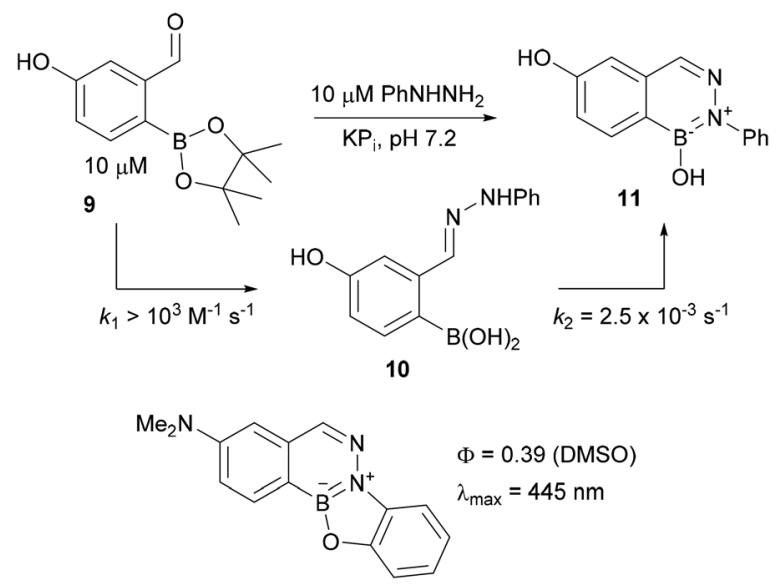

Fig. 3 Top: Borazaroisoquinolines form irreversibly when hydrazine derivatives are condensed with 2-FPBA. The reaction's modularity and the formation of an extended aromatic structure can be exploited to create fluorescent derivatives. where compartmentalization could keep systems out of equilibrium it would be ideal to have an irreversible linkage. Work from my group ${ }^{33}$ as well as Susan Bane's lab $^{35}$ has recently identified an irreversible variant in the hydrazone series (see Fig. 3). It turns out that when hydrazines are condensed with 2-FPBA the hydrazone adducts undergo a secondary cyclization reaction to create aromatic borazaroisoquinolines (BIQs, see structure $\mathbf{1 1}$ in Fig. 3). BIQs are robust and their formation is irreversible. $^{36}$ The extended aromatic ring created upon BIQ formation opens the door to modulating its photophysical properties - indeed we have exploited this feature to create a blue fluorescent turn-on fluorophore (see bottom of Fig. 3). Although in chemical biology red and near-infrared fluorophores are in greater need than blue ones, the ability to build a turn-on fluorophore in such short order is a promising start towards creating more practical variants.

\section{Future outlook}

The next major step for boron-assisted oxime ligations or BIQ formations is to prove they can operate in vivo with high efficiency. We have already shown that the reaction is unaffected when run in cell lysates and in the presence of common biological interfering agents, ${ }^{4}$ but the area of greatest unmet need in chemical biology is in in vivo chemical ligations. We are currently working on this problem.

\section{Notes and references}

1 E. M. Sletten and C. R. Bertozzi, Angew. Chem., Int. Ed., 2009, 48, 6974-6998.

2 F. Saito, H. Noda and J. W. Bode, ACS Chem. Biol., 2015, 10, 1026-1033.

3 D. M. Patterson, L. A. Nazarova and J. A. Prescher, ACS Chem. Biol., 2014, 9, 592-605.

4 P. Schmidt, C. Stress and D. Gillingham, Chem. Sci., 2015, 6, 3329-3333.

5 A. Bandyopadhyay and J. Gao, Chem. - Eur. J., 2015, 21, 14748-14752.

6 A. Darko, S. Wallace, O. Dmitrenko, M. M. Machovina, R. A. Mehl, J. W. Chin and J. M. Fox, Chem. Sci., 2014, 5, 3770-3776.

7 A. Sachdeva, K. Wang, T. Elliott and J. W. Chin, J. Am. Chem. Soc., 2014, 136, 7785-7788.

8 G. T. Hermanson, Bioconjugate techniques, Elsevier/Academic Press, Amsterdam, 2008.

9 W. P. Jencks, in Progress in Physical Organic Chemistry, John Wiley \& Sons, Inc., 1964, pp. 63-128.

10 E. H. Cordes and W. P. Jencks, J. Am. Chem. Soc., 1962, 84, 826-831.

11 A. Dirksen, T. M. Hackeng and P. E. Dawson, Angew. Chem., Int. Ed., 2006, 45, 7581-7584.

12 A. Dirksen and P. E. Dawson, Bioconjugate Chem., 2008, 19, 2543-2548. 
13 Y. Zeng, T. N. C. Ramya, A. Dirksen, P. E. Dawson and J. C. Paulson, Nat. Methods, 2009, 6, 207-209.

14 K. Lang and J. W. Chin, ACS Chem. Biol., 2014, 9, 16-20.

15 C. Uttamapinant, A. Tangpeerachaikul, S. Grecian, S. Clarke, U. Singh, P. Slade, K. R. Gee and A. Y. Ting, Angew. Chem., Int. Ed., 2012, 51, 5852-5856.

16 C. Uttamapinant, M. I. Sanchez, D. S. Liu, J. Z. Yao and A. Y. Ting, Nat. Protocols, 2013, 8, 1620-1634.

17 D. C. Kennedy, C. S. McKay, M. C. B. Legault, D. C. Danielson, J. A. Blake, A. F. Pegoraro, A. Stolow, Z. Mester and J. P. Pezacki, J. Am. Chem. Soc., 2011, 133, 17993-18001.

18 N. Devaraj and R. Weissleder, Acc. Chem. Res., 2011, 44, $816-827$.

19 N. K. Devaraj, R. Weissleder and S. A. Hilderbrand, Bioconjugate Chem., 2008, 19, 2297-2299.

20 N. Devaraj, R. Upadhyay, J. Haun, S. Hilderbrand and R. Weissleder, Angew. Chem., Int. Ed., 2009, 48, 7013-7016.

21 N. Devaraj, S. Hilderbrand, R. Upadhyay, R. Mazitschek and R. Weissleder, Angew. Chem., Int. Ed., 2010, 49, 28692872.

22 V. Hong, S. I. Presolski, C. Ma and M. G. Finn, Angew. Chem., Int. Ed., 2009, 48, 9879-9883.

23 S. I. Presolski, V. Hong, S.-H. Cho and M. G. Finn, J. Am. Chem. Soc., 2010, 132, 14570-14576.

24 M. Hutin, G. Bernardinelli and J. R. Nitschke, Chem. - Eur. J., 2008, 14, 4585-4593.
25 Y. Pérez-Fuertes, A. M. Kelly, A. L. Johnson, S. Arimori, S. D. Bull and T. D. James, Org. Lett., 2006, 8, 609612.

26 R. Nishiyabu, Y. Kubo, T. D. James and J. S. Fossey, Chem. Commun., 2011, 47, 1124-1150.

27 D. A. Tickell, M. F. Mahon, S. D. Bull and T. D. James, Org. Lett., 2013, 15, 860-863.

28 P. M. S. D. Cal, J. B. Vicente, E. Pires, A. V. Coelho, L. s. F. Veiros, C. Cordeiro and P. M. P. Gois, J. Am. Chem. Soc., 2012, 134, 10299-10305.

29 N. J. Gutierrez-Moreno, F. Medrano and A. K. Yatsimirsky, Org. Biomol. Chem., 2012, 10, 6960-6972.

30 A. Bandyopadhyay, K. A. McCarthy, M. A. Kelly and J. Gao, Nat. Commun., 2015, 6, 6561.

31 A. Bandyopadhyay and J. Gao, J. Am. Chem. Soc., 2016, 138, 2098-2101.

32 J. Crugeiras, A. Rios, E. Riveiros and J. P. Richard, J. Am. Chem. Soc., 2009, 131, 15815-15824.

33 C. J. Stress, P. J. Schmidt and D. G. Gillingham, Org. Biomol. Chem., 2016, 14, 5529-5533.

34 A. Adamczyk-Woźniak, K. M. Borys, I. D. Madura, A. Pawełko, E. Tomecka and K. Żukowski, New J. Chem., 2013, 37, 188-194.

35 O. Dilek, Z. Lei, K. Mukherjee and S. Bane, Chem. Commun., 2015, 51, 16992-16995.

36 M. J. S. Dewar and R. C. Dougherty, J. Am. Chem. Soc., 1964, 86, 433-436. 